\title{
A participar se aprende participando. VdB: Acción y reflexión críticas en el proyecto para la regeneración urbana participativa en el barrio Virgen de Begoña (Madrid)
}

\author{
Paisaje Transversal: Guillermo Acero Caballero, Jon Aguirre Such, Jorge Arévalo Martín, Pilar Díaz \\ Rodríguez e Iñaki Romero Fernández de Larrea*
}

\section{Resumen}

Hasta la fecha, la regeneración urbana ha ido asociada a gestiones costosas e inversiones económicas elevadas, que equivalen a largos procesos de desarrollo de las intervenciones o a dificultades para ponerlas en marcha. Frente a esta realidad, que debilita la implicación ciudadana, planteamos una metodología capaz de implicar a la ciudadanía y de desarrollar estrategias parciales con visión integral que supongan menor gasto y mayor efectividad. Desarrollamos procesos participativos que detecten necesidades integrales de un barrio sobre las que proponer intervenciones puntuales de regeneración que tengan impactos integrales: prototipos.

Planteamos una estructura organizativa de los procesos de regeneración urbana participativos con el objetivo de facilitar su replicabilidad. Esta se configura a través de tres canales, tres estrategias paralelas en el tiempo pero transversales en el contenido. Cada una de ellas persigue varias metas que se complementan y que, en conjunto, abordan el proyecto de modo integral: Difusión, Ciudadanía y Proyecto participativo.

Paisaje Transversal, equipo transdisciplinar compuesto por profesionales en red con expertos de diversas disciplinas, y dedicado a investigar y proponer nuevos modelos de gestión urbana y de transformación del entorno construido, ha desarrollado y puesto en marcha la metodología descrita en el contexto del barrio Virgen de Begoña.

\section{Palabras clave}

Agricultura Urbana; Huertos Comunitarios; Regeneración Urbana; Participación Ciudadana; Urbanismo Participativo

\section{Abstract: Participating teaches how to participate. VdB: Critical action and reflection on the project for participative urban regeneration in the district of Virgen de Begoña (Madrid).}

To date, urban regeneration has been associated with costly management and high economic investment, which have equated to long project development processes or to start-up difficulties. Faced with this reality, which weakens citizen involvement, a methodology is proposed that is capable of involving the citizenry and developing partial strategies with a holistic vision which represent greater cost effectiveness. Participative processes are developed which detect the comprehensive needs of a district upon which to propose specific regeneration interventions that have comprehensive impact: prototypes.

An organizational structure of participative urban regeneration processes is proposed with the objective of facilitating its reproducibility. This is configured through three channels, three strategies parallel in time but transverse in content. Each of them pursues several complementary goals which, together, approach the project in a comprehensive manner: Diffusion, Citizenry and Participative Project.

Paisaje Transversal, a trans-disciplinary team composed of a network of expert professionals from diverse disciplines, dedicated to investigating and to proposing new models of urban management and transformation of the built surroundings, has developed and put into effect the described methodology in the context of the Virgen de Begoña district. 


\section{Key words}

Urban Agriculture; Community Gardens; Urban Regeneration; Citizen Participation; Participatory Urbanism

\section{Introducción: La participación ciudadana ante el cambio de época}

"Las ideas son el resultado de los hechos, y no los hechos de las ideas, y el pueblo no será libre cuando deje de ser inculto, sino que dejará de ser inculto cuando sea libre". John Berger. G. (1972)

Vivimos un momento histórico de cambio de época. Nuestras pautas de vida y de trabajo se han transformado, también lo han hecho la manera en la que nos comunicamos y nos relacionamos. Por no hablar de las estructuras familiares, las cuales ya no responden a los cánones tradicionales en las que la cabeza familiar marcaba un referente jerárquico incuestionable. Todos estos parámetros describen la base sobre la que comienza a construirse un consenso cada vez más amplio acerca de la necesidad de reconfiguración de las estructuras políticas y sociales tradicionales; e Internet, las redes sociales virtuales y las tecnologías de la información y comunicación (TIC) están desempeñando un papel decisivo en el desarrollo de esta nueva conciencia colectiva (Subirats, 2011).

Tal y como indica acertadamente Mark Poster, Internet no es un nuevo "martillo" que sirve para clavar más rápido los "clavos" de siempre. Internet no es el fax. Es un invento que está transformando radicalmente la forma en la que los seres humanos nos comunicamos, nos relacionamos o nos asociamos. En la red se generan dinámicas sociales que se reflejan en la esfera física, lo que produce una alteración de las pautas sociales que, a su vez, se reflejan en la esfera virtual. De este modo, se genera un bucle de retroalimentación que define un espacio relacional híbrido, sobre el que se está formalizando un inminente cambio de paradigma sociopolítico. En este sentido, Internet seguramente se pueda asemejar a la imprenta o la máquina de vapor de nuestro tiempo, más que a un nuevo "martillo", pues plantea un escenario colectivo en el que se establecen nuevas formas de relacionarse y de vivir. A través de Internet recobramos el debate sobre lo común, gracias a los canales de movilización, debate y transferencia de conocimiento colectivo y horizontal que ha generado. Con Internet estamos aprendiendo a desarrollar nuevas capacidades que permiten una mayor autonomía entre la población, y que han de tenerse en cuenta a la hora de plantear la nuevas formas de gestión e intervención en la ciudad y el territorio.

Esta introducción no pretende ser un alegato procibernético ni mucho menos. Simplemente es un intento de reflejar el contexto actual sobre el que nos va a tocar intervenir; si no lo conocemos, no podremos establecer estrategias participativas eficaces que se hagan eco de este nuevo escenario social. No podemos permanecer ajenos a estos condicionantes, pues abren un nuevo y estimulante campo de acción en el que muchos de los preceptos válidos hasta el momento han de ser reformulados. Los nuevos retos que se nos plantean requieren no solo nuevas herramientas, sino un profundo cambio en las estructuras de pensamiento. No podemos afrontar los retos del nuevo siglo con esquemas mentales y metodologías de trabajo del siglo $\mathrm{XX}^{1}$.

Además, la irrupción de las nuevas tecnologías virtuales coincide con un hastío y una desconfianza crecientes de la población hacia la clase política. En España, la irrupción del 15M, un movimiento ciudadano "rizomático" que está constituyéndose en un nuevo agente político, es un claro ejemplo (¿consecuencia?) de ello. No en vano,

\footnotetext{
${ }^{1}$ "Se requieren nuevas formas de pensar para resolver los problemas creados por las viejas formas de pensar" Albert Einstein.
} 
detrás del famoso lema "¡No nos representan!" hay mucho más que un amplio descontento social frente a las instituciones y los partidos políticos. Bajo él subyacen unas ganas renovadas de participar e involucrarse en la política de lo cotidiano, en las decisiones que configuran nuestro entorno, en la respuesta conjunta a los problemas colectivos, así como en el establecimiento de redes de apoyo mutuo que permitan introducir mejoras en nuestro hábitat. La esfera local se fortalece y se conecta gracias a los avances tecnológicos con lo global; $y$ es precisamente en esta tensión entre lo hiperlocal y lo hiperglobal donde podemos encontrar las claves del cambio de paradigma en la práctica urbanística y en los procesos participativos.

A pesar de la clara deslegitimación de instituciones, partidos, sindicatos y demás estructuras políticas que hasta el momento el momento han capitalizado los canales de participación "democrática", paralelamente estamos observando cómo la Administración muestra una sensibilidad cada vez mayor hacia la participación ciudadana. Ya sea por intereses políticos o como mero ejercicio cosmético, lo cierto es que cada vez son más las instituciones públicas que impulsan, con mayor o menor acierto, procesos participativos. Por ello, convendría interpretar este "mantra" participativo como una oportunidad que nos permita realmente desarrollar prácticas y experiencias que avalen la idea de que a través de la participación ciudadana los proyectos urbanos son mejores y más eficaces.

Evidentemente, no conviene ser ilusos en este campo y pensar que las personas al frente de los poderes públicos van a decidir ahora, súbitamente, ceder su parcela de poder a la ciudadanía; y hay que ser conscientes de que, muchas veces, los procesos participativos no son más que objetos propagandísticos vacíos de contenido. Pero también es verdad que hoy en día existe una intención "aperturista" cada vez mayor por parte de los organismos públicos que técnicos y ciudadanos tenemos que saber aprovechar y canalizar en aras del bien común.

Para ello tenemos que empezar a generar experiencias que avalen esa necesidad de hacer partícipe e implicar a la comunidad en el desarrollo de los proyectos que pretendan modificar su entorno próximo. Tenemos que ser capaces de demostrar, a través de proyectos concretos, que los proyectos desarrollados a través de la participación ciudadana no solo son necesarios, sino posibles y mejores. No obstante, para producir este cambio de paradigma en el urbanismo, no necesitamos únicamente "buenas prácticas" o procesos exitosos, sino, además, una nueva mentalidad y una nueva teoría para poder afrontarlos.

Vivimos y pensamos las ciudades desde una perspectiva fuertemente enraizada en una teoría que debe actualizarse. Es el momento de avanzar sobre estas bases y desarrollar nuevas formas y vías de intervenir en el territorio. Si comprendemos que Internet altera la forma en la que interactuamos las personas, en la que muchas de las lógicas de la esfera virtual modifican las pautas sociales de la esfera física y viceversa; que transforma los procesos de intermediación estableciendo un marco conceptual y relacional más horizontal; que genera vínculos mucho más directos e inmediatos; y que supone, en consecuencia, una intensificación de las relaciones personales, podemos aceptar que estamos ante un profundo cambio en nuestras sociedades. Por ende, la manera en la que se piensan los procesos participativos tendrá que adaptarse también a esta evolución.

No obstante no conviene olvidar todas las experiencias históricas que se han producido en España que ponen en relación urbanismo y participación ciudadana, que han permitido establecer un sólida base teórica y práctica sobre la que pensar estos nuevos modelos de construcción 
democrática de la ciudad. Conviene, por tanto, revisar las exitosas experiencias de movilización y participación social surgidas en el ocaso de los años setenta y cuyo desarrollo coincidió con la primera década de la democracia española, hasta prácticamente los años noventa. El ímpetu de aquel movimiento vecinal iniciático, que contaba con el asesoramiento técnico de urbanistas y profesionales relacionados con las prácticas urbanas, marcó la agenda política del último cuarto del siglo XX. Esta relación entre ciudadanos y técnicos fructificó en la transformación y recualificación de las tramas urbanas de muchos barrios populares, gracias a las generosas inversiones públicas. Sin duda todos estos proyectos constituyen un referente a la hora de encarar cualquier proyecto de regeneración integrada en claves participativas, si bien han de ser actualizados en desde una perspectiva contemporánea.

Es por ello que en Paisaje Transversal hacemos nuestra la sentencia de John Berger que citábamos al principio, y creemos que la mejor manera de desarrollar buenas ideas o teorías es a través de la acción; pero no cualquier tipo de acción: una acción reflexiva que en primera instancia venza la consabida muletilla de "la parálisis por el análisis", para posteriormente replegarse sobre sí misma y plantear momentos más pausados en los que producir pensamiento crítico. No hay teoría posible sin acción, y no hay acción sin teoría. Tenemos que ser capaces de generar unos bucles en los que práctica y pensamiento se retroalimenten constantemente. Para nosotros son variables de una misma ecuación, pero no por ello nos enclaustramos en una biblioteca. Es importante desarrollar estrategias de emprendimiento urbano.

Lo que a continuación os relatamos no es sino el producto de cuatro años dedicados a la reflexión —nacimos como blog de reflexión crítica en torno a la ciudad, el urbanismo, el territorio y el paisaje en 2007: www.paisajetransversal.org - y cómo posteriormente hemos aplicado todo el pensamiento desarrollado durante este tiempo en proyectos concretos durante aproximadamente un año y cómo, a su vez, este artículo nos ha servido para replantearnos a nosotros mismos y nuestras acciones.

De este modo, primero describiremos en forma de glosario una serie de conceptos que nos parecen relevantes a la hora de pensar en la ciudad y el urbanismo desde una perspectiva contemporánea; posteriormente, describiremos la metodología propia que hemos desarrollado -la cual es totalmente copyleft y cualquiera puede apropiarse de ella-y, finalmente, relataremos cómo hemos aplicado todas estas cuestiones de manera práctica en proyectos concretos.

\section{Los conceptos: Del bottom-up a la beta permanente}

Como ya sabemos, en las últimas décadas, en las políticas urbanas españolas ha primado el crecimiento expansivo, con el suelo como principal recurso de financiación municipal, en perjuicio de la intervención en los tejidos ya consolidados. EI actual contexto económico ha puesto de manifiesto la imposibilidad de mantener dichas dinámicas, lo que supone una oportunidad para trasladar nuestro ámbito de trabajo hacia los entornos urbanos consolidados y su mejora. La ciudad sobre la que nos tocará intervenir en los próximos años no es la "nueva ciudad", un espacio definido por las exigencias normativas y las necesidades inmobiliarias, sino la ciudad consolidada: aquella cuyas transformaciones urbanas solo se producen cuando la población entiende como propios los cambios; aquella en la que conceptos básicos como la cohesión social o la sostenibilidad adquieren un significado efectivo. Esta situación, sin embargo, también pone de manifiesto la necesidad de nuevos mecanismos de acción.

En primer lugar, la inclusión del tejido ciudadano en los procesos de decisión se muestra no solo 
como un condicionante ético, sino como una necesidad básica para que los esfuerzos políticos y económicos resulten efectivos. La realidad ciudadana, que podía ser obviada en los procesos de nuevos barrios periféricos por la sencilla razón de que no contaban con residentes previos, se muestra sumamente relevante en los tejidos consolidados. El componente histórico de estos entornos, que a menudo fueron construidos y ocupados en períodos relativamente cortos y que aún a día de hoy mantienen características comunes, ha de contemplarse no como un impedimento, sino como un valor potencial capaz de otorgar cohesión a cada uno de los barrios.

\section{Bottom-up}

Surge así el concepto de proyectos bottom-up (de abajo arriba), procesos en los que el tejido social es el impulsor del proyecto, y que surgen y se estructuran desde la base social. Cabe entender estos proyectos no como procesos ajenos a la Administración y a sus cauces legales, sino como mecanismos de apropiación y empoderamiento, en los que los ciudadanos entienden como propio un proceso de transformación del que se sienten partícipes. Frente a los procesos top-down (de arriba abajo), la lógica bottom up permite dinamizar comunidades desde pequeñas parcelas de acción, haciendo énfasis en las pruebas tempranas, como estrategia para comprobar la eficiencia de las partes. El reto que la lógica bottom-up planteará será el de conjugar estas pequeñas parcelas de acción con los objetivos a largo plazo, de manera que los avances conseguidos reviertan de manera eficaz en proyectos integrales futuros.

Una lógica bottom-up, entendida como proceso de transformación urbana, precisa de la inclusión no solo del tejido ciudadano, sino de todos los actores presentes directa o indirectamente en el entorno. La ciudad hiperconectada en la que vivimos deslocaliza en sumo grado a dichos actores (diversas escalas de la Administración Pública, agentes locales, instituciones, empresas privadas y grupos informales), cuya situación de incomunicación dificulta enormemente los procesos. Por ello, la capacidad de todos los agentes para participar de manera activa en estos procesos pasa por la existencia de estructuras organizativas consolidadas, ya sean formales o informales, y de canales de comunicación y diálogo que permitan un intercambio fluido.

\section{Espacios de comunicación y diálogo}

A este respecto, la dificultad de la administración para establecer canales de diálogo con la ciudadanía y con el resto de agentes, así como el hermetismo al proceder en los proyectos, ha penalizado el éxito de muchos procesos de regeneración urbana. Una comunicación fluida desde el inicio del proceso y una preocupación real por su transparencia podría resolver muchos de los problemas que surgen al concebir la participación de manera meramente justificativa 0 , en el mejor de los casos, consultiva. Por ello, es necesario establecer canales de comunicación entre una ciudadanía activa y la Administración Pública, que superen las dinámicas reivindicativas y conviertan, así, las demandas y reclamaciones ciudadanas en estrategias propositivas, capaces de aportar soluciones al tiempo que se visibilizan los problemas.

\section{Facilitadores}

En este contexto, el ámbito profesional ha de reconsiderar su papel desde una perspectiva colaborativa. Las condiciones de partida anteriormente citadas ponen de manifiesto la necesidad de equipos que desarrollen la labor de interlocución y facilitación de los procesos, y garanticen la comunicación entre los actores. La figura del facilitador emerge como agente externo que promueve los procesos, capaz de poner en diálogo perspectivas divergentes y a menudo contra- 
puestas, transformar las reclamaciones en estrategias propositivas y poner en marcha herramientas y canales de diálogo nuevos que agilicen los procesos. La capacidad técnica de dichos equipos permitirá traducir la información ciudadana en propuestas técnicas, generar confianza entre los diversos actores y dar respuestas creativas a las distintas actividades, propuestas y acciones que se vayan a desarrollar.

El equipo profesional encargado del proceso que actúe como facilitador llevará a cabo principalmente las siguientes tareas:

- Interlocución imparcial entre ciudadanos y políticos.

- Coordinación de las distintas áreas de gobierno implicadas en el proceso.

- Gestión de las herramientas digitales de comunicación para facilitar la inclusión de todos los agentes y mantener la transparencia con los ciudadanos durante el proceso.

- Gestión de bases de datos de información ciudadana y su visualización online y analógica. La información analógica se gestionará en colaboración con las asociaciones de vecinos.

- Documentación de los procesos, mediante la materialización de las necesidades y las propuestas en documentos dirigidos a los distintos técnicos de las áreas de gobierno involucradas.

\section{Espacios híbridos}

Ante nosotros se abre un campo de experimentación absolutamente estimulante, en el que tendremos que empezar a plantear estrategias profesionales estrechamente ligadas y permeables a todas las lógicas sociales y tecnológicas que definen nuestro tiempo. La evolución —o revolución- tecnológica de las últimas décadas ha alterado profundamente nuestra manera de relacionarnos entre nosotros y con nuestro entorno.
Al tiempo, las políticas aplicadas en relación con el espacio público han ido paulatinamente socavando su capacidad para funcionar como espacio de socialización, decisión y resolución de conflictos. En este sentido, la esfera digital se ha revelado como un espacio público más activo y participativo que el espacio público tradicional, que ha quedado relegado, en el mejor de los casos, a una mera función de representación. Uno de los retos durante los próximos años será alcanzar la convergencia entre ambas esferas, la digital y la física, con el objetivo de generar una ciudadanía más crítica y proactiva. Los nuevos mecanismos de participación deberán desarrollarse de manera coordinada en el espacio digital y en el espacio físico, en lo local y en lo global, a través de estrategias capaces de obtener un mayor grado de representatividad en los procesos de decisión.

Para ello tendremos que aplicar una nueva lógica enraizada en el pensamiento en red y en las nuevas estrategias provenientes de la esfera digital. Estas estrategias tendrán que permitir agilizar y democratizar los procesos de decisión, así como incorporar las redes sociales dentro de las estrategias participativas, no como sustitución de los procesos en el entorno físico, sino como consecución de espacios de trabajo híbridos, producto de un viaje de ida y vuelta entre ambas esferas. A este respecto, el concepto de brecha digital se ve complementado a día de hoy con los llamados nativos digitales, individuos o colectivos que tienen totalmente interiorizados el lenguaje y los códigos de la tecnología digital. Así, la idea de mediación queda ampliada al ámbito de lo digital.

\section{Beta permanente}

Dentro de este ámbito, resulta interesante recoger el concepto de beta permanente, entendido no solo como un estado puntual inmaduro, sino como un proceso de mejora continua necesario para la experimentación y la evolución. "Beta permanente" hace alusión a la reivindicación del 
proceso frente al objeto -lógica procesual frente a lógica objetual- a la horizontalidad del trabajo y al pensamiento en red (network thinking), al desarrollo de la inteligencia social y colectiva, a la apropiación comunitaria de los proyectos, a la colaboración y a las redes de apoyo mutuo.

Aplicar la lógica de beta permanente pasa por entender los proyectos urbanos como procesos abiertos, dinámicos y en constante evolución, en los que el objeto final no es determinado a priori. Se trata de establecer puntos de partida y horizontes de posibilidades. De este modo, la formalización de la intervención final es decidida colectivamente como resultado de la interacción entre los actores implicados y el conocimiento que cada uno de ellos aporta. Para lograr esta conjunción de saberes resulta imprescindible empezar a generar y estructurar procesos participativos de calidad, que no son otros que los que permiten la apropiación de los proyectos urbanos por parte de los ciudadanos.

\section{Dinámicas colaborativas}

La teoría de juegos, un área de las matemáticas aplicadas a los procesos de toma de decisión, abrió la puerta a opciones que van más allá de los sistemas basados en la competencia entre participantes. Los llamados juegos cooperativos o de suma no cero favorecen dinámicas en las que la ganancia de unos no implica la pérdida de otros. De esta manera, el concepto de diseño colaborativo plantea procesos de creación en los que varios agentes trabajan juntos en pos de metas comunes, combinando los conocimientos y los recursos de todos ellos. Al operar de esta manera se trata de incentivar y aprovechar la inteligencia colectiva cotidiana para la optimización de los diseños generados. Al mismo tiempo, las dinámicas colaborativas se constituyen a día de hoy como mecanismos más adaptables y resilientes que los tradicionales procesos de decisión jerarquizados; esto permite dar respuestas más eficaces e innovadoras, especialmente en situaciones en las que los implicados son múltiples y heterogéneos (Figura 1).

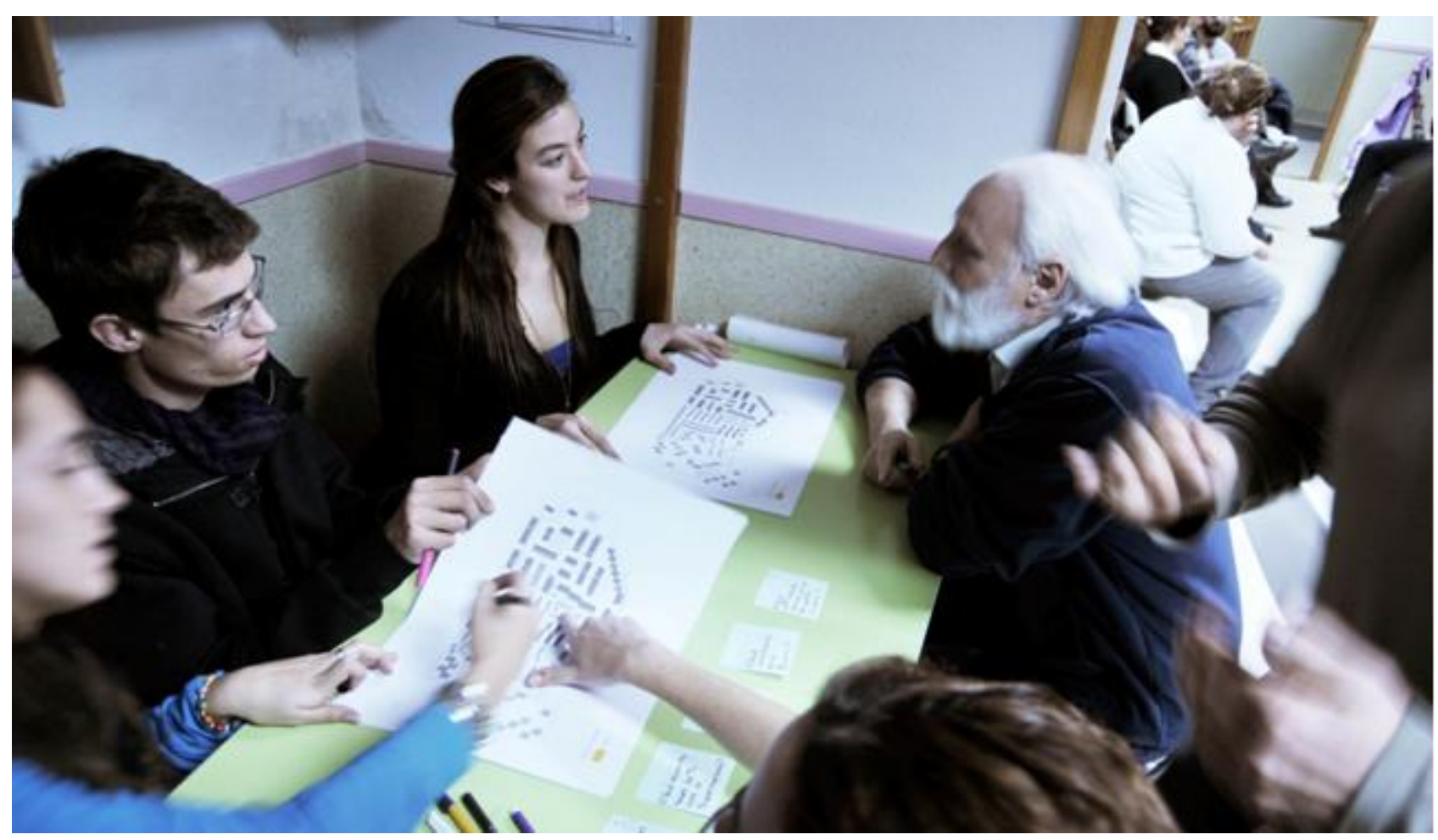

Figura 1. "Facilitadores del diseño ciudadano".Taller de diseño colaborativo entre vecinos del barrio Virgen de Begoña (Madrid) y alumnos del Instituto Europeo di Design. Fuente: Elaboración propia. 
Por otra parte, las dinámicas colaborativas entran directamente en la línea de flotación del cambio de época que reseñábamos al principio. Se trata, por tanto, de entender que las nuevas lógicas sociales comienzan a atender mucho más a la colaboración entre individuos que a la competición entre ellos. De esta manera, existe un aprecio cada vez mayor por lo común -lo que algunos denominan "el procomún"-, y esta preocupación está produciendo unas pautas sociales que rompen con la lógica competitiva del capitalismo para establecer nuevos escenarios colaborativos en los que la posesión prima sobre la propiedad, y en los que la generación de valor sin competir es ajena a la lógica de coste-beneficio o la monetarización de los proyectos. En este sentido, en la lógica de lo común, del bien común, lo que prevalece es la cooperación y lo relacional, frente a la competición y lo transaccional.

\section{Pequeño proyecto-grandes proyectos}

La necesidad de generar mecanismos resilientes y adaptables surge de un contexto urbano donde los cambios se producen cada vez a mayor velocidad. Las transformaciones urbanas, al menos desde el ámbito más reglamentado, se desarrollan en función de figuras de planeamiento que, por su constitución, presentan dificultades para adaptarse a los cambios que se producen en la ciudad. En los últimos años, la regeneración urbana se ha asociado a gestiones costosas e inversiones económicas elevadas, que se traducen en largos procesos de desarrollo de las intervenciones $y$ en dificultades para ponerlos en marcha. Frente a esta realidad, que debilita la implicación ciudadana, los procesos participativos bottom-up tienen la capacidad de detectar necesidades y establecer líneas estratégicas sobre las que proponer intervenciones puntuales que tengan impactos integrales.

Cuando trabajamos en objetos definitivos, optamos por adoptar aproximaciones sucesivas, de bajo coste y modificables, que cristalicen en intervenciones capaces de evolucionar por sí solas. Esta manera de proceder implica operar dentro de la lógica del testeo o del desarrollo de productos críticos no definitivos, como sucesivas aproximaciones a la intervención óptima; es decir, generar dispositivos con los que los ciudadanos puedan interactuar, comprobando si se ajustan a sus necesidades y expectativas, y acompasando el proceso participativo con las intervenciones físicas. Esto permite extraer conclusiones con el objetivo de adaptar y mejorar las intervenciones posteriores, planteando las transformaciones urbanas como procesos más continuos que discretos.

Las estrategias parciales dentro de proyectos integrales pueden modificarse y readaptadarse para definir con mayor efectividad la solución adecuada para cada caso. Estos "prototipos" abren posibilidades de replicabilidad que, asociadas al bajo coste de las intervenciones, permiten extender las actuaciones a un mayor número de barrios, beneficiando por tanto a un mayor número de ciudadanos. El principal reto a largo plazo será gestionar eficazmente la evaluación y el seguimiento de las distintas estrategias parciales, de modo que se puedan incorporar en la estrategia global.

\section{Metodología DCP: Difusión, Ciudadanía y Proyecto participativo}

\section{Pertinencia}

Incluso desde una perspectiva participativa, el proyecto urbano convencional se revela insuficiente para resolver los problemas a los que se enfrenta la ciudad hoy en día. La redefinición del papel del técnico pasa por diversificar nuestra labor más allá del desarrollo de proyectos de intervención, incorporando todos los subprocesos previos que garantizan un desarrollo eficaz. El proyecto como tal ha de complementarse con 
estrategias de visibilización, concienciación y pedagogía, y de apropiación por parte de los ciudadanos. De nada sirve desarrollar un proyecto si primero no somos realmente conscientes de los conflictos que hemos de resolver, si no somos capaces de implicar a los ciudadanos en organizaciones lo suficientemente representativas o si no se ha desarrollado una labor de pedagogía profunda.

Ante esta situación, planteamos una metodología capaz de fomentar la implicación ciudadana, que se desarrolla a través de estrategias parciales englobadas en una visión integral, que supongan mayor eficacia. De este modo, se trazan procesos participativos orientados hacia la detección de necesidades de un barrio, sobre las que proponer intervenciones puntuales de regeneración a través de prototipos.

De entre los distintos beneficios asociados a los procesos participativos para el prototipado de intervenciones, destacamos los siguientes:

- Por su escala, las intervenciones parciales requieren menor inversión económica que la reurbanización completa de un barrio, lo que permite extender las actuaciones de regeneración a más barrios, y así beneficiar a un mayor número de ciudadanos.

- La facilidad de ejecución de algunas de las actuaciones permite acompasar el proceso participativo con las intervenciones físicas. Con ello se consigue una mayor satisfacción de la ciudadanía durante el proceso y, por consiguiente, su implicación y participación.

- Resiliencia: El desarrollo a lo largo del proceso de intervenciones puntuales permite probar los beneficios de cada intervención; esto, a su vez, permite extraer conclusiones para adaptar y mejorar las intervenciones posteriores, y así conseguir una respuesta más eficaz a la continua transformación de la ciudad.

\section{Metodología}

El desarrollo de la metodología que planteamos tiene como objetivo definir ciertos protocolos revisables en lo que respecta a la regeneración urbana participativa, para que sean fácilmente replicables. La configuración de esta metodología atiende a tres canales, tres estrategias paralelas en el tiempo pero transversales en el contenido. Cada una de ellas persigue varias metas complementarias que, en conjunto, abordan el proyecto desde una perspectiva integral: Difusión (D), Ciudadanía (C) y Proyecto participativo (P).

(D) El canal Difusión proyecta tanto la visibilización local y global de las propuestas como su transparencia, principalmente a través de las posibilidades que ofrecen las redes sociales y las nuevas herramientas digitales de difusión, aunque sin olvidar los medios de comunicación tradicionales.

En proyectos integrales, la difusión consigue ampliar el colectivo local comprometido con el proyecto y, gracias a la repercusión exterior, genera un intercambio de impresiones global, enriqueciendo así las propuestas. En segundo lugar, la transparencia que ofrece la difusión se traduce en una herramienta de presión colectiva que supervisa el cumplimiento y la consecución de las propuestas tal y como fueron planteadas.

Aplicaciones prácticas: Creación de identidades digitales (perfiles en diferentes redes sociales virtuales) sobre los proyectos para visibilizar y supervisar los procesos; creación de blogs y perfiles en otras redes sociales como Twitter o Facebook para difundir contenidos y generar retornos en la red, de manera que se enriquezcan las propuestas con las aportaciones externas; y generar una imaginería (logotipos, eslóganes, diseños, etc.) con la que la comunidad se sienta identificada, como estrategia para facilitar la implicación y apropiación ciudadana.

Actuaciones tipo: Gestión de redes sociales y 
blogs, creación de plataformas georreferenciadas de información y difusión entre foros profesionales como charlas, jornadas o foros online, publicaciones, reportajes audiovisuales, etc.

(C) El canal Ciudadanía trabaja la concienciación respecto a la participación, el espacio público o la ecología, así como la identidad comunitaria a través de la pedagogía y la información.

De esta manera se consigue generar una estructura social en la que queda reforzada la identidad comunitaria respecto al espacio urbano. Especialmente, se potencia el movimiento colaborativo, los valores del espacio público, los principios de sostenibilidad y el potencial de las herramientas digitales para facilitar los procesos.

Aplicación práctica: Programación de actividades lúdicas y de sensibilización (talleres, charlas, asambleas, reuniones, juegos, etc.) que permitan generar una pedagogía urbana entre la población. Actuaciones tipo: Eventos populares, sensibilización sobre espacio público, ecología y participación a través de los colegios, centros de mayores o centros sociales, actividades, talleres o estrategias para fomentar la identidad local, etc.

(P) El canal Proyecto participativo se ocupa de proponer modelos de gestión y diseños urbanos transdisciplinares y participativos de forma local y global, a través de dos fases solapables: el diagnóstico propositivo y participativo y las propuestas piloto, para concluir con la propuesta final integral. Las propuestas piloto son estructuras flexibles, fácilmente programables, desprogramables y reprogramables. Constituyen en sí pequeñas pruebas de laboratorio (urbano) que sirven para someter a prueba las soluciones de modo temporal, evitando con ello poner en práctica la solución final de forma definitiva, lo que conllevaría una mayor inversión económica sin una garantía de éxito. Para poder aplicarse, el canal Proyecto participativo requiere los otros dos canales.

Aplicación práctica: Diseño y ejecución de intervenciones puntuales de carácter revisable en función de los efectos que produzcan. Estas acciones deben estar englobadas dentro de un proyecto y una perspectiva integrales que aportará el equipo técnico que desarrolla la propuesta. No se trata tanto de un parche momentáneo, sino de acciones precisas englobadas en un marco integral más amplio. Por otra parte, el diseño y, cuando las circunstancias lo precisen, la ejecución de dichas actuaciones se realizarán en colaboración con la comunidad.

Actuaciones tipo: Actividades de diagnóstico colaborativo como video-mapeos, talleres de diseño colaborativo con ciudadanos, universitarios y técnicos, ejecución de intervenciones efímeras, etc.

Si bien los tres canales discurren paralelos en el tiempo, su peso específico varía a lo largo del proceso. En las fases iniciales, los canales de Difusión y Ciudadanía tienen mayor importancia con el objetivo de ampliar el colectivo implicado, dar visibilidad al proceso y generar mayor identidad y cohesión social. A medida que avanza el proceso, el canal Proyecto participativo ha de cobrar importancia para articular los procesos de decisión y priorizar las intervenciones (Figura 2). A continuación os presentamos $\mathrm{VdB}$, un proyecto desarrollado en el barrio Virgen de Begoña (Madrid), en el que Paisaje Transversal hemos aplicado esta metodología. 

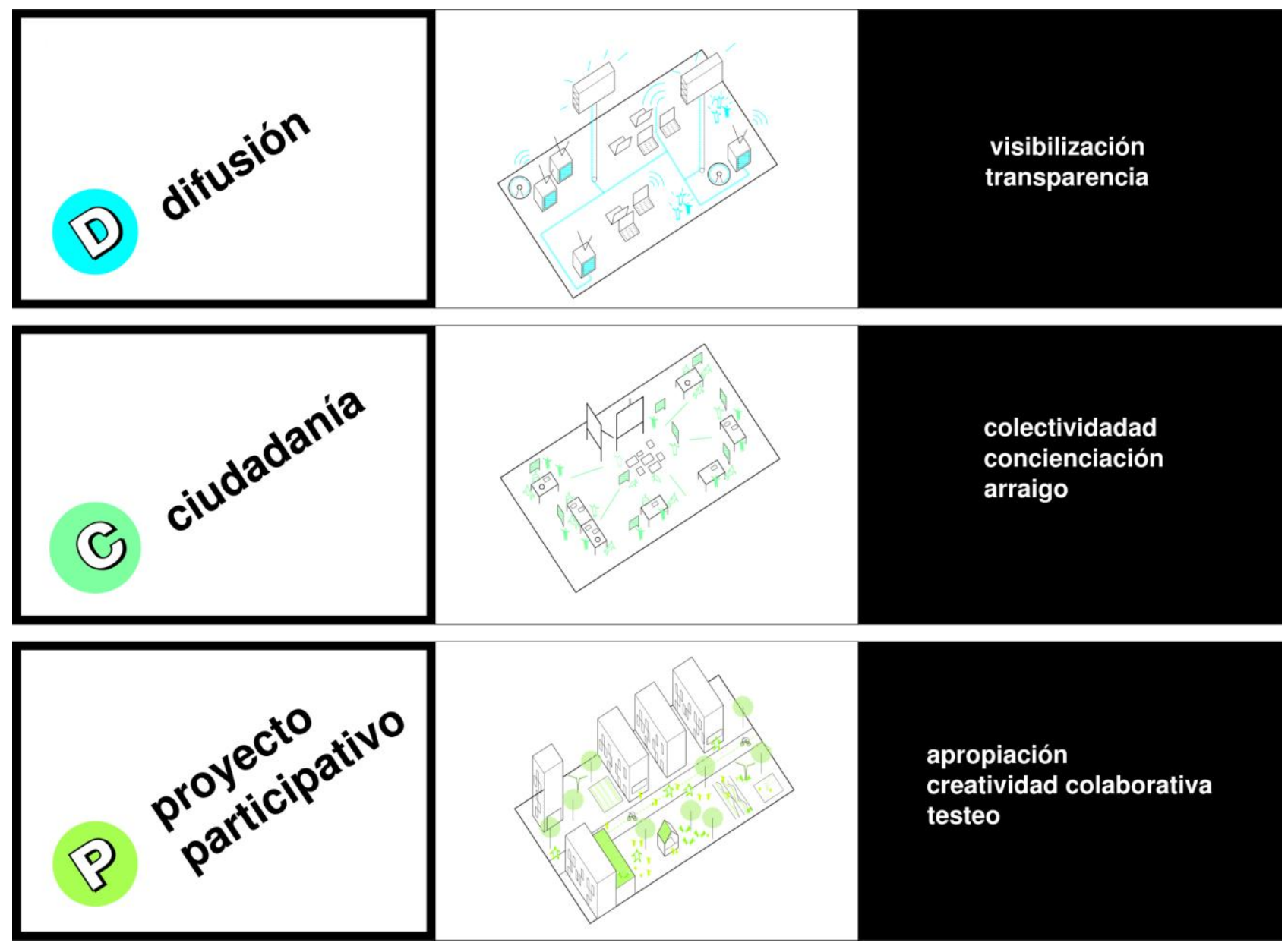

Figura 2. Metodología DCP. Difusión, ciudadanía y proyecto participativo. Fuente: Elaboración propia.

\section{VdB: Experiencia bottom-up para la regeneración urbana integral y participativa en el barrio Virgen de Begoña (Madrid)}

La colonia Virgen de Begoña constituye un ejemplo de los más de doscientos polígonos de bloque abierto que se han detectado en Madrid como zonas de intervención prioritaria por su actual estado de deterioro ${ }^{2}$. Situado en el distrito de Fuencarral-El Pardo, junto al nudo norte de la M30 , se presenta rodeado por las infraestructuras de la A-1, la M-607 y la propia M-30, así como por las vías del ferrocarril en su borde norte, lo que intensifica su condición de isla con respecto a las

2“ El Programa municipal de actuación para la periferia: Análisis de los Polígonos en Bloque abierto, desarrollado por Área de Gobierno de Urbanismo, Vivienda e Infraestructuras del Ayuntamiento de Madrid, es un interesante análisis general de esta "periferia intermedia".trabaje de otro modo." (Lerner, 2003) tramas colindantes (véanse Figura 3 y Cuadro 1). Este hecho no hace sino ahondar las vulnerabilidades que presenta el barrio a día de hoy, muchas de ellas comunes al resto de los polígonos de bloque abierto de esta "periferia intermedia": obsolescencia e inadecuación de la edificación, escasa calidad de la urbanización y baja identidad del espacio público, una estructura demográfica y socioeconómica debilitada y oportunidades limitadas para la actividad económica por tratarse de tejidos eminentemente monofuncionales. En el caso concreto de Virgen de Begoña, el Plan Parcial Prolongación de la Castellana introduce nuevas incógnitas en las condiciones del tejido, situándolo en una nueva posición de centralidad poco acorde con su estado real de deterioro. 


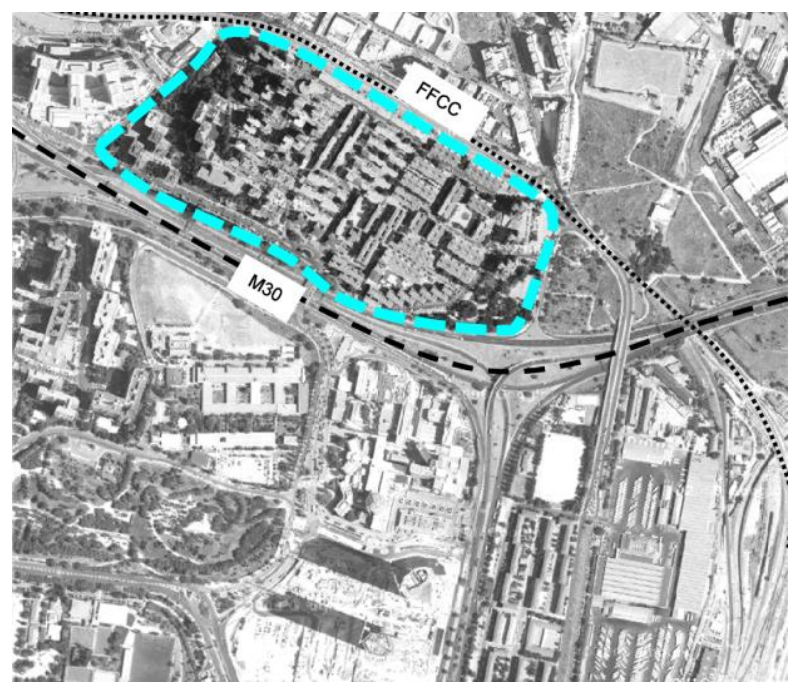

Figura 3. Fotografía aérea de la colonia Virgen de Begoña. Las infraestructuras circundantes (M-30, vías del tren y el hospital Ramón y Cajal) le confieren un fuerte carácter de "isla". Fuente: Elaboración propia.

Información general del barrio Virgen de Begoña

\begin{tabular}{|l|l|}
\hline Población & 8163 habitantes \\
\hline Antigüedad del polígono & 1961 \\
\hline Superficie polígono $\left(\mathrm{m}^{2}\right)$ & 186614 \\
\hline Número de viviendas & 3642 \\
\hline Densidad (viviendas/Ha) & 195,16 \\
\hline Número de edificios & 218 \\
\hline
\end{tabular}

Cuadro 1. Información general del barrio Virgen de Begoña. Fuente: Ayuntamiento de Madrid.

Este proyecto quiere recuperar y actualizar los históricos procesos de regeneración urbana participativa que se desarrollaron durante los años ochenta en el área metropolitana de Madrid. En aquella época se impulsó un contundente programa de reforma urbanística coincidiendo con la redacción del Plan General de 1985, que propició la práctica total erradicación del chabolismo y la infravivienda, así como la construcción de equipamientos y espacios públicos de calidad. Casos paradigmáticos como el de la Meseta de Orcasitas o el Pozo del Tío Raimundo marcan un precedente para la labor que Paisaje Transversal está desarrollando en esta colonia de la periferia intermedia madrileña.

\section{Desarrollo del proyecto}

El proceso de Virgen de Begoña se origina en febrero de 2011, cuando tiene lugar el acercamiento por parte del equipo de Paisaje Transversal a la Asociación de Vecinos de Begoña, con el propósito de iniciar un proceso participativo para fortalecer la identidad comunitaria y dar respuesta a las demandas vecinales que se venían presentando ante la Junta de Distrito, concernientes a los problemas de movilidad, accesibilidad y contaminación acústica que afectan al barrio. En este momento, la labor se limita al apoyo de las iniciativas vecinales, de carácter fundamentalmente reivindicativo, y a reuniones informales que sirven como acercamiento a las reclamaciones vecinales y generan la confianza necesaria para iniciar el proceso. Comienzan entonces las labores de difusión a través de la red, que permiten visualizar la problemática existente, combinadas con iniciativas que refuerzan la identidad comunitaria. A este respecto, se lleva a cabo la creación de un blog (<http://vdebegona.wordpress.com $>$ ) como medio de supervisión del proyecto y punto de encuentro digital con los habitantes del barrio. Las siglas "VdB", que crean una imagen fácilmente reconocible, se constituyen en concepto como el primer bien de uso común surgido en el contexto del proceso, generado en un primer momento por el equipo de Paisaje Transversal. Su gran repercusión interna y externa facilitó que los propios vecinos se identificasen con el proyecto, condición previa a su apropiación posterior.

Se inicia entonces el período de presentación del proyecto VdB: se dan a conocer las posibilidades de llevar a cabo un proyecto bottom-up (impulsado desde la base social) frente al procedimiento habitual en el que la decisión procede de la Administración (top-down), para lo cual resulta necesario alcanzar un mayor grado de representatividad y de implicación vecinal en los procesos de toma de decisiones. Una primera presentación a 
través de la Asociación de Vecinos pone de manifiesto la buena disposición de estos para embarcarse en un proyecto de estas características. El conocimiento del largo plazo y la manera en que este se gestiona será uno de los puntos esenciales de la estrategia futura. Por otro lado, la necesidad de extender el proyecto más allá del tejido asociativo tradicional dará paso a la segunda etapa del proyecto, en la que, a través de la recuperación de las fiestas del barrio, se revelará el verdadero potencial de la iniciativa.
El proceso de acercamiento y generación de confianza de la primera etapa debe ser complementado con acciones propositivas, capaces de dar respuesta a las necesidades identificadas hasta el momento. La etapa de empoderamiento se inicia a través del taller vecinal "Recuerdos y Deseos" (Figura 4), dinámica participativa que dirige la mirada simultáneamente a la realidad pasada y a las oportunidades futuras, combinando las potencialidades de uno y otro.

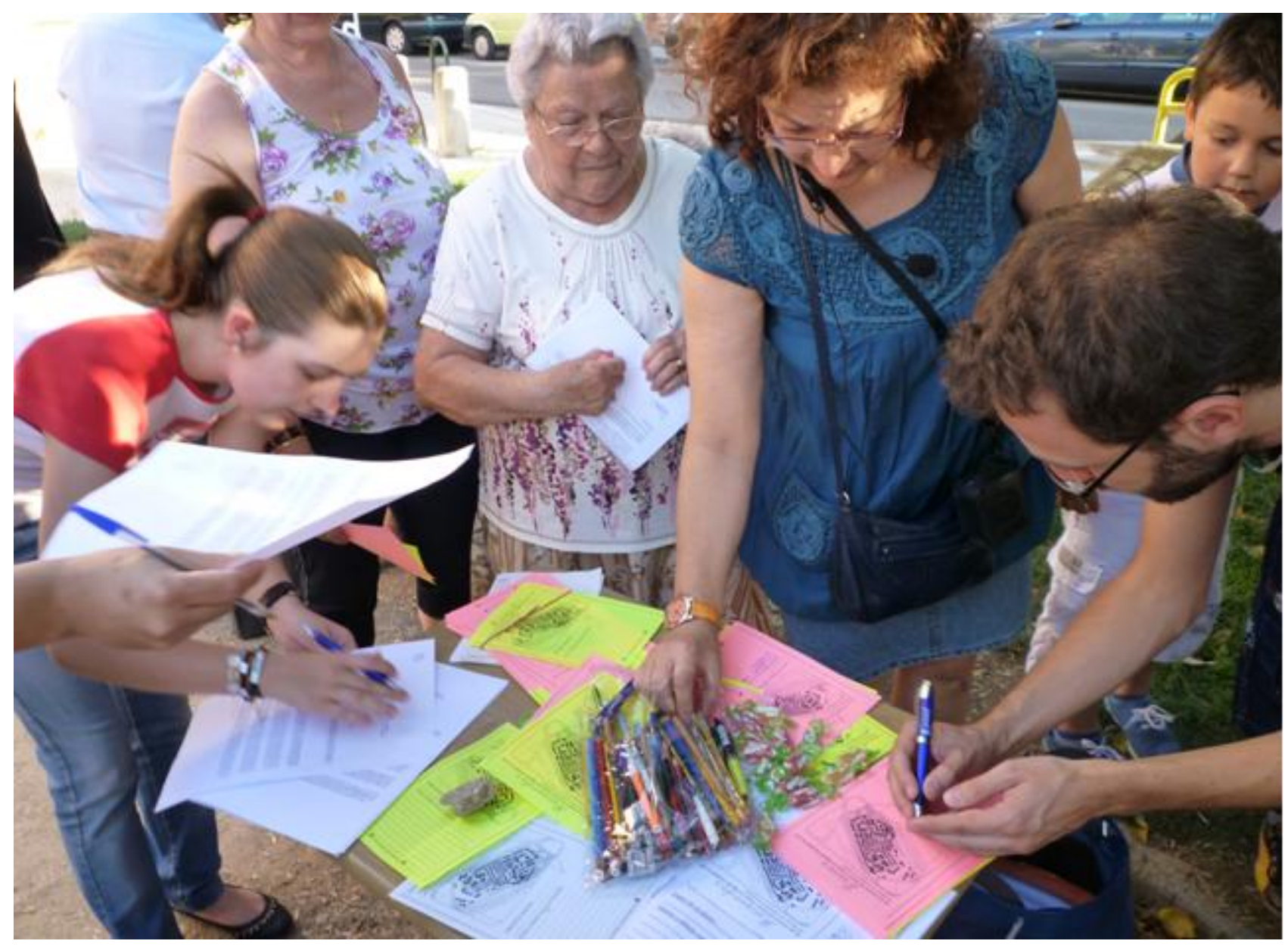

Figura 4. Taller “Recuerdos y Deseos”. Fuente: Elaboración propia.

De los resultados extraídos de este taller surge la idea de recuperación de las fiestas del barrio, perdidas hace más de treinta años. De esta manera, el proceso de empoderamiento cristaliza definitivamente a través de la "Fiestacción", concebida como evento lúdico de carácter reivindicativo capaz de extender $\mathrm{VdB}$ al mayor número de vecinos. Durante el proceso de preparación del evento emerge un grupo motor, el primer organismo informal surgido dentro del propio proyecto y que a día de hoy se mantiene operativo. Se trata de un grupo que aglutina miembros de la Asociación de Vecinos, personas ajenas a ésta y otras que posteriormente pasan a formar parte de la Asociación. De esta manera, la Asociación de Vecinos y el grupo motor se entienden como 
estructuras organizativas compatibles, capaces de desarrollar labores complementarias. La conformación de este grupo y las labores de difusión desarrolladas con motivo de la "Fiestacción" consiguen ampliar y diversificar el alcance de la iniciativa, tanto en la dimensión digital como en la material (véase Figura 5). En lo que respecta a esta última, la presencia de miembros jóvenes en el grupo facilita el acercamiento a los colegios del barrio, y, así, la implicación de los alumnos en el evento a través de la realización colectiva de murales, que posteriormente serán expuestos durante la fiesta. La combinación de este y otros esfuerzos, llevados a cabo por el grupo motor, la Asociación de Vecinos y Paisaje Transversal, da como resultado un evento de actividades múltiples, capaz de atraer a los diferentes sectores de población del barrio, con especial énfasis en el juvenil e infantil, por su capacidad para atraer al resto.

El éxito de la "Fiestacción" marca el inicio del proceso en su vertiente propositiva. Hasta ese momento, los aspectos de difusión y ciudadanía habían resultado prioritarios y necesarios: constituían la base sobre la que empezar a construir. Una vez asentados los cimientos y tras la recogida de información a través de las actividades realizadas, las reuniones posteriores permiten consensuar las líneas estratégicas básicas sobre las que discurrirá el proyecto. La necesidad de potenciar el uso del espacio público, hecho que la "Fiestacción" había dejado patente, desemboca en la línea estratégica de intervención en los espacios interbloque, una de las principales deficiencias del tejido, y al mismo tiempo, el gran potencial del barrio. La mejora en el ámbito de la diversidad de actividades, como solución a su condición de barrio dormitorio, conforma la línea estratégica de reactivación económica. Finalmente, la situación de aislamiento que sufre con respecto a su entorno próximo plantea la estrategia de bordes urbanos como otra de las necesidades primarias.

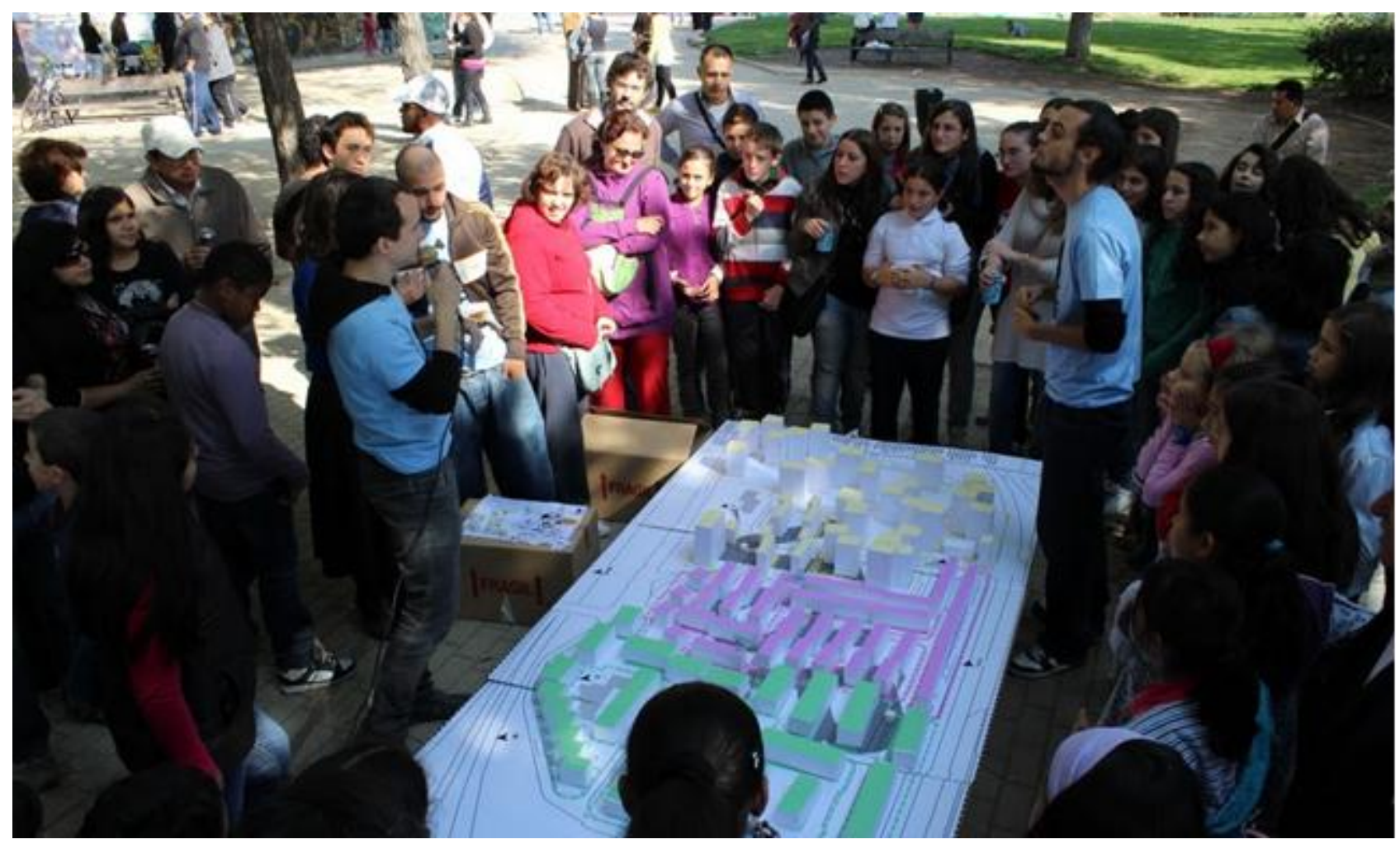

Figura 5. Imagen de la "Fiestacción" durante el desarrollo del juego de mesa gigante "Trivial de VdB", una actividad lúdico-pedagógica sobre estrategias para la regeneración urbana del barrio. Fuente: Elaboración propia. 
La definición de líneas estratégicas que posibiliten intervenciones parciales con visión integral abre un nuevo marco de actuación que va más allá de los mecanismos tradicionales para la rehabilitación urbana integral. Con respecto a esto, la línea estratégica de Reactivación Económica se inicia a través de actividades de mapeo colectivo, combinando las vertientes física y digital. Por su parte, la estrategia de intervención en el Espacio Interbloque se ha desarrollado a través de talleres de diseño colaborativo entre vecinos y técnicos, en un proceso que actualmente sigue en marcha.

La capacidad para estructurar dichas acciones a través de actividades que puedan asumir los diferentes organismos públicos (administraciones, universidades, fundaciones, etc.) responde no solo a la coyuntura económica actual, sino también a la realidad social de una ciudadanía que ha de mantenerse implicada en procesos que pueden dilatarse en el tiempo. Por otro lado, la inclusión de los diferentes organismos públicos permite, más allá de diversificar las cargas económicas, hacer partícipes en el proceso a todos los agentes implicados. De esta forma, se introducen los prototipos intersectoriales: procesos participativos que detecten las necesidades integrales de un barrio sobre las que proponer proyectos puntuales de intervención capaces de dar respuestas a las problemáticas más acusadas desde una perspectiva integral. Esto se lleva a cabo a través de la vinculación transversal de las acciones que se establezcan como prioritarias con los programas de actuación y presupuestos correspondientes a las distintas áreas de gobierno. Asimismo, resulta fundamental avanzar hacia fórmulas de cooperación entre lo público y lo privado a la hora de financiar y ejecutar algunas actuaciones, mediante el establecimiento de sinergias en las que prevalezcan de manera innegociable los intereses del bien común.

\section{Fases del proyecto}

El proceso tal y como se ha desarrollado hasta la fecha puede dividirse en tres fases:

\section{Fase 1. Duración: 5 meses}

Canales: Difusión (D) y Ciudadanía (C)

Actividades desarrolladas:

- Difusión y visibilización de las iniciativas vecinales.

- Actividades lúdico-pedagógicas: Taller "Recuerdos y Deseos", "Fiestacción”, etc.

- Recogida de información cuantitativa y cualitativa procedente de la ciudadanía y de los estudios ya realizados por la Administración.

- Toma de contacto con la Junta de Distrito y otros agentes del barrio (comerciantes, asociaciones culturales, etc.).

Objetivos:

- Generación de confianza hacia los diferentes actores.

- Visibilización del proceso entre los vecinos.

- Ampliación del colectivo implicado.

- Validar y ajustar los estudios de diagnóstico con la información obtenida de los vecinos a través de las actividades.

- Implicación de la Junta de Distrito FuencarralEl Pardo en el proceso, de cara a su activación como coordinador a nivel institucional.

\section{Fase 2. Duración: 8 meses}

Canales: Difusión (D) y Ciudadanía (C) y Proyecto participativo $(\mathrm{P})$

Actividades desarrolladas:

- Definición de líneas estratégicas sobre las que actuar y programa de actividades en torno a ellas:

- Espacio interbloque.

- Bordes urbanos.

- Reactivación económica: Actividades de análisis comercial y planteamiento de estrategias ("Video-mapeo streaming").

- Apertura a agentes privados externos 
(universidades, fundaciones, etc.) para desarrollar actividades sobre las líneas estratégicas definidas. Taller "Facilitadores del diseño colaborativo" con alumnos del Instituto Europeo di Design de Madrid.

- Contacto con áreas de gobierno del Ayuntamiento de Madrid y estudio de los programas existentes que permitan intervenir en el barrio.

- Exposición del proyecto en congresos y foros profesionales (5. CONAMA Local, Congreso INTA35, Seminario Final proyecto Rehabitat, Festival eme 3 Bottom Up, etc.).

- Publicación de artículos en revistas especializadas (Revista Urban-e, Ciudad Sostenible, etc.).

Objetivos:

- Comprobación del grado de aceptación de las propuestas.

- Implicación de las áreas de gobierno pertinentes.

- Visibilización del proyecto en el ámbito profesional.

- Fijación de prioridades entre las líneas de trabajo en función de su capacidad de incidencia en el barrio y de las posibilidades establecidas con las áreas de gobierno.

Fase 3. En desarrollo

Canales: Difusión (D) y Ciudadanía (C) y Proyecto participativo $(\mathrm{P})$

Actividades

- Elaboración de la documentación para coordinar los diferentes programas y las áreas de gobierno implicadas.

- Eventos de visibilización de las intervenciones.

- $\quad$ Talleres de diseño colaborativo.

- Desarrollo de actividades correspondientes a las líneas de trabajo a largo plazo, con el fin de desarrollar las labores de pedagogía necesarias.

Objetivos:

- Implicación de los vecinos del barrio en las intervenciones que se desarrollen.

- Consecución de mejoras en el barrio que mantengan o amplíen el grado de participación.

- Coordinación de las áreas de gobierno correspondientes.

- Creación de mecanismos de colaboración público-privado para las intervenciones y la gestión de estas.

Paisaje Transversal es una plataforma que trabaja en nuevos modelos colaborativos de gestión e intervención urbana desde el punto de vista participativo, transdisciplinar y ecológico, facilitados por el uso de las nuevas herramientas digitales (<http://www.paisajetransversal.org>).

\section{Referencias}

BERGER, John. G. Madrid: Alfaguara, 1994.

BORJA, Jordi. Ciudadanía y espacio público. Barcelona: CCCB, 1998.

DELEUZE, Gilles y GUATTARI, Félix. Mil Mesetas (capitalismo y esquizofrenia). (6. ${ }^{\mathrm{a}} \mathrm{ed}$ ). Valencia: Pre-Textos, 2004.

GOULD, Stephen Jay. La vida maravillosa. Barcelona: Editorial Crítica, 2006.

LÓPEZ DE LUCIO, Ramón. Construir ciudad en la periferia. Madrid: Mairea Libros, 2007.

LORENZO, Ana Rosa y MARTíNEZ, Miguel. Asambleas y reuniones. (2. ${ }^{\text {a }}$ ed). Madrid: Traficantes de Sueños, 2005.

MARTÍN, Tomás: Del barro al barrio. Madrid: Asociación de Vecinos de la Meseta de Orcasitas, 1997.

NAVARRO, Clemente. Participatory democracy and political opportunism: municipal experience in Italy and Spain (1960-93). International Journal of Urban and Regional Research, 2004, vol. 
28, № 4, p. 819-838.

SUBIRATS, Joan: Otra sociedad, ¿otra política?

Barcelona: Icaria editorial, 2011.

VILLASANTE, Tomás R. (Ed.). Retrato de chabolista con piso. Madrid: CIDUR S.A, 1989.

\section{Cita del artículo}

PAISAJE TRANSVERSAL. A participar se aprende participando. VdB: Acción y reflexión críticas en el proyecto para la regeneración urbana participativa en el barrio Virgen de Begoña (Madrid). Hábitat y Sociedad, 2012, no 4, p. 15-31. <www.habitatysociedad.us.es>.

http://dx.doi.org/10.12795/HabitatySociedad.2012.i4.02 